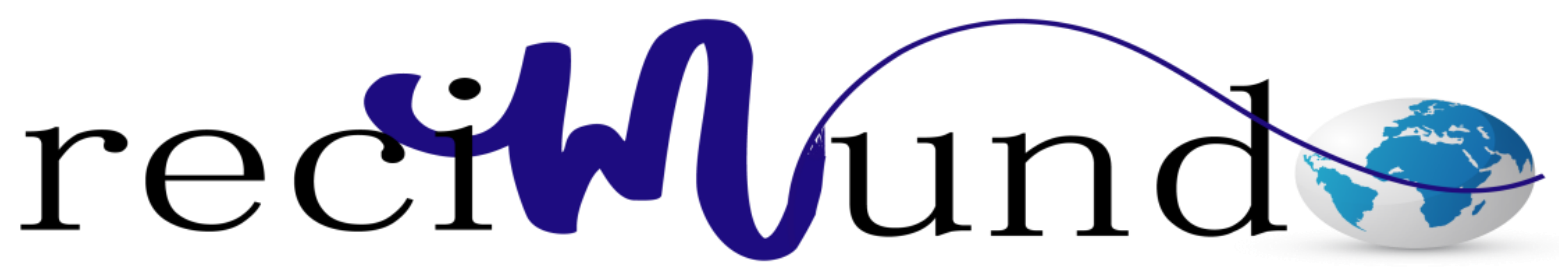

Revista Científica Mundo de la Investigación y el Conocimiento

Carol Yacqueline Muso Pilchisaca ${ }^{a}$; Alex Christopher Ruiz Arriciaga ${ }^{\text {b }}$; Cristhian Javier Córdova Molina ${ }^{c}$; Mariuxi Jacqueline Coronel Reinoso ${ }^{d}$

Factores que influyen en la aparición de dermatomiositis como enfermedad autoinmune

Factors that influence the appearance of dermatomyositis as an autoimmune disease

Revista Científica Mundo de la Investigación y el Conocimiento. Vol. 2 núm.3, julio, ISSN: 2588-073X, 2018, pp. 510-526

DOI: 10.26820/recimundo/2.(3).julio.2018.510-526

Editorial Saberes del Conocimiento

Recibido: 05/04/2018

Aceptado: 15/02/2018

Publicado: 30/07/2018

Correspondencia: msp.cmuso@gmail.com
a. msp.cmuso@gmail.com
b. alexruiza3@gmail.com
c. criscordovam@hotmail.com
d. mcoronelreinoso@gmail.com 


\section{Factores que influyen en la aparición de dermatomiositis como enfermedad autoinmune}

Vol. 2, núm. 3., (2018)

Muso Pilchisaca Carol Yacqueline; Alex Christopher Ruiz Arriciaga; Cristhian Javier Córdova Molina; Mariuxi Jacqueline Coronel Reinoso

\section{RESUMEN}

Como enfermedad inmunológica, la dermatomiositis debe prestársele importancia médica dado que no presenta factor especifico de su aparición, esta miopatía se caracteriza por lesiones en la piel de color purpura, debilidad muscular y fiebre. En cuanto a su frecuencia, pueden considerarse dentro del grupo de enfermedades raras debido a su baja incidencia, la afectación principal es a nivel de piel y músculos, con las variantes de ser un proceso agudo, sub- agudo y crónico, pudiendo ocasionar atrofia y degeneración muscular. El objetivo de investigar acerca de esta enfermedad es con la finalidad de analizar de forma descriptiva la bibliografía del o los factores que inciden en la aparición de la dermatomiositis como enfermedad autoinmune, para el diagnóstico a tiempo. El diagnóstico de la enfermedad se aprecia a través de exploración hospitalaria donde se realizan evaluaciones clínicas, pruebas de laboratorio de rutina, histopatologías y tomando en cuenta los indicadores de Bohan y Peter. Estos científicos incluyen las particularidades propias de la enfermedad tales como: debilidad muscular proximal y simétrica, elevación de enzimas musculares, cambios electromiográficos típicos de miositis, rash característico, biopsia muscular demostrando miositis inflamatoria. Se concluye que, es una miopatía inflamatoria de gran repercusión en la sociedad, lo cual deriva en la significación de efectuar estudios sobre la misma donde pueda generarse información acerca de su incidencia.

Palabras Claves: Dermatomiositis, Enfermedad, Autoinmune, Diagnóstico, Debilidad Muscular. 


\title{
Factores que influyen en la aparición de dermatomiositis como enfermedad autoinmune
}

Vol. 2, núm. 3., (2018)

Muso Pilchisaca Carol Yacqueline; Alex Christopher Ruiz Arriciaga; Cristhian Javier Córdova Molina; Mariuxi Jacqueline Coronel Reinoso

\begin{abstract}
As an immunological disease, dermatomyositis should be given medical importance since it does not have a specific factor for its incidence, this myopathy is characterized by purple skin lesions, muscle weakness and fever. Regarding their frequency, they can be considered within the group of rare diseases due to their low incidence, the main affectation is at the level of skin and muscles, with the variants being an acute, sub-acute and chronic process, being able to cause atrophy and muscular degeneration. The objective of investigating this disease is to analyze in a descriptive way the bibliography of the factors that affect the appearance of dermatomyositis as an autoimmune disease, for timely diagnosis. The diagnosis of the disease can be seen through a hospital examination where clinical evaluations, routine laboratory tests, histopathologies and taking into account the indicators of Bohan and Peter are carried out. These scientists include the particular characteristics of the disease such as: proximal and symmetric muscle weakness, muscle enzyme elevation, electromyographic changes typical of myositis, characteristic rash, muscle biopsy demonstrating inflammatory myositis. It is concluded that it is an inflammatory myopathy of great repercussion in society, which results in the significance of carrying out studies on it where information about its incidence can be generated.
\end{abstract}

Keywords: Dermatomyositis, Disease, Autoimmune, Diagnosis, Muscular Weakness. 


\section{Factores que influyen en la aparición de dermatomiositis como enfermedad autoinmune}

Vol. 2, núm. 3., (2018)

Muso Pilchisaca Carol Yacqueline; Alex Christopher Ruiz Arriciaga; Cristhian Javier Córdova Molina; Mariuxi Jacqueline Coronel Reinoso

\section{Introducción.}

La dermatomiositis constituye una enfermedad inmunológica que afecta el movimiento del individuo al contraerse y relajarse ocasionando la inflamación de los músculos, así como alteración cutánea (piel) y cuyas causas de origen son poco conocidas.

La enfermedad suele presentarse a cualquier edad de la persona, generalmente se exterioriza en adultos a partir de los 40 años hasta los 60 años aproximadamente, también es visible en los niños durante su etapa juvenil, es decir en las edades de 05 a 15 años. Afecta a más mujeres que hombres. Dicha enfermedad inmunológica, actualmente es vista como una amenaza de salud pública dado que se le asocia con factores infecciosos, endocrinos, genéticos y ambientales, así como con la formación anormal de algún tejido con posible representación tumoral; la dermatomiositis como enfermedad inmunológica ha sido poco diagnosticada, sin embargo según la revisión bibliográfica en la Revista Médica Hondureña y a criterio de Fernández (1967) se puede mencionar un estudio realizado por el Dr. Carlos A. Delgado, del Servicio de Pediatría del Hospital San Felipe, Honduras quien en 1953, “sospechó en una niña la presencia de una Dermatomiositis”.

A nivel mundial diversos estudios llevados a cabo en diversos puntos del globo donde se establecen una incidencia anual media de 2,1 a 7,7 casos nuevos por millón de habitantes y año (Amato, 2009). Según Villa -Juarez (2002), la dermatomiositis “puede presentarse en niños entre 5 -14 años, con un pico más frecuente alrededor de los 50 años”. Asimismo en España Mastaglia (2002) señala que "la incidencia anual media es similar, de 2,2 a 10,6 casos nuevos por millón de habitantes y año" (p.198). 


\section{Factores que influyen en la aparición de dermatomiositis como enfermedad autoinmune}

Vol. 2, núm. 3., (2018)

Muso Pilchisaca Carol Yacqueline; Alex Christopher Ruiz Arriciaga; Cristhian Javier Córdova Molina; Mariuxi Jacqueline Coronel Reinoso

De acuerdo a Bohan y Peter (1975) la enfermedad se diagnostica en aspectos que incluyen "debilidad muscular, rash cutáneo característico (rash en heliotropo o pápulas de Gottron sobre superficie extensora de articulaciones de dedos, codos, rodillas y tobillos), enzimas musculares elevadas, cambios miopáticos en la electromiografía, biopsia con alteraciones características, en ausencia de otras causas de miopatías". Asimismo los autores señalan que "la presencia del compromiso de piel característico es fundamental para el diagnóstico. La debilidad muscular se encuentra casi siempre presente” (p.101).

La justificación de este artículo es relevante en el campo medico dado que en las pocas publicaciones bibliográficas que se tienen de este tema, se muestra que los factores que desencadenan esta enfermedad son aún desconocidos, por lo tanto, la dermatomiositis en un caso inmune muy raro, donde no se tiene determinado a ciencia cierta ¿cuál o cuáles son los factores que influyen para la aparición de dermatomiositis?; en tal sentido el objetivo de esta investigación se centra en analizar de forma descriptiva la bibliografía del o los factores que inciden en la aparición de la dermatomiositis y con ello desarrollar mecanismos para su conocimiento, que podrían aportar acciones prácticas en el área de salud para hacerle frente a esta enfermedad autoinmune que va en aumento.

\section{Método.}

En virtud de desarrollar la temática abordada, se consultó diversos medios documentales, donde se efectuó la búsqueda de literatura pertinente a la enfermedad dermatomiositis, muy especialmente las fuentes de búsqueda a través de herramientas desarrolladas por las tecnologías de información y comunicación (Tic) que facilitan la difusión y acceso a publicaciones 


\section{Factores que influyen en la aparición de dermatomiositis como enfermedad autoinmune}

Vol. 2, núm. 3., (2018)

Muso Pilchisaca Carol Yacqueline; Alex Christopher Ruiz Arriciaga; Cristhian Javier Córdova Molina; Mariuxi Jacqueline Coronel Reinoso

científicas de valor y rigurosidad académica; realizado esto se tomo en cuenta las fuentes documentales digitales que aportan información acerca de los factores que inciden en la aparición de la dermatomiositis, síntomas, causas, evaluaciones, tratamiento.

En tal sentido, la información recolectada acerca del tema, precisó seleccionar y organizar material escrito para su comprensión, seguidamente efectuar lecturas para realizar un subrayado de las ideas principales de las publicaciones elegidas, se analizó la información efectuando un resumen bibliográfico, posteriormente se procedió a dar una explicación textual analítica coherente y significativa del tema. Por lo tanto, mediante estas acciones se logrará analizar detalladamente la bibliografía del o los factores que inciden en la aparición de la dermatomiositis, aspectos que permitirán la posibilidad de generar información reveladora actualmente escasa y poco conocida en la incidencia de la enfermedad.

\section{Resultados.}

\section{La Dermatomiositis: Definición}

Es una enfermedad que se manifiesta a través de diversos factores con evidentes características dermatológicas. Dalakas (2003) considera que la dermatomiositis "es una enfermedad inflamatoria que afecta a la piel y el músculo. Se incluye dentro de las miopatías inflamatorias idiopáticas o miositis idiopáticas, que son un grupo heterogéneo de enfermedades musculares de etiología desconocida que se caracterizan por la aparición progresiva de debilidad muscular e inflamación". 


\section{Factores que influyen en la aparición de dermatomiositis como enfermedad autoinmune}

Vol. 2, núm. 3., (2018)

Muso Pilchisaca Carol Yacqueline; Alex Christopher Ruiz Arriciaga; Cristhian Javier Córdova Molina; Mariuxi Jacqueline Coronel Reinoso

En esta dirección la dermatomiositis según Vidarte (2008) es una "miositis inflamatoria caracterizada por la aparición de pródromos imprecisos, edema, dermatitis e inflamación y degeneración muscular. Es una afección relativamente rara, 2 veces más frecuente en mujeres de 40 a 65 años de edad que en hombres". (p. 576).

Por su parte Peloro (2001) indica que la dermatomiositis juvenil "es una enfermedad multisistémica de etiología desconocida, con inflamación de los músculos y la piel por compromiso de vasos pequeños, como arteriolas y capilares. Constituye la miopatía inflamatoria idiopática más frecuente en la niñez”. (p. 405).

De acuerdo con los autores consultados, se interpreta que la dermatomiositis constituye un padecimiento de índole inflamatorio que involucra el tejido cutáneo y muscular del individuo, y cuya causa de origen es desconocida, clasificada como una enfermedad autoinmune de rara. La enfermedad aparece con síntomas de debilidad muscular, muy especialmente en mujeres de entre 40-60 años de edad, no obstante en la etapa de niñez y adolescencia también puede tener presencia; por tal motivo puede inferirse que la dermatomiositis es de carácter autoinmune que se presenta tanto en adultos como niños.

\section{Clasificación}

La dermatomiositis es una enfermedad catalogada como rara (Dalakas, 2003). Afecta a niños y adultos, desarrollándose más en mujeres (relación 2:1), (Jacobson, 1997). Posee un carácter inmunopatológico que provoca inflamación y destrucción de fibras musculares y cutáneas. A criterio de Gerami (2006) la relación “mujer:hombre es de 2-3:1” (p.602). Existen 


\section{Factores que influyen en la aparición de dermatomiositis como enfermedad autoinmune}

Vol. 2, núm. 3., (2018)

Muso Pilchisaca Carol Yacqueline; Alex Christopher Ruiz Arriciaga; Cristhian Javier Córdova Molina; Mariuxi Jacqueline Coronel Reinoso

varias propuestas de clasificación, mismas que han venido cambiando conforme a los avances tecnológicos en el área de la salud. Bohan y Peter en 1975 fueron los primeros en dividirla según su clínica en polimiositis (PM), dermatomiositis (DM), PM o DM asociada a cáncer, DM de la infancia y PM o DM asociada a una colagenopatía.

Autores como Bielsa-Marsol (2009) y Hassan (2009) a su vez subdividieron las formas infantojuvenil y del adulto en dos grupos: clásica y clínicamente amiopática, de acuerdo con la presencia o ausencia de sintomatología muscular. Por su parte Mastaglia (2002) integra propuestas anteriores de Karpati (1988) y Dalakas (1991), donde se tiene:

I. Dermatomiositis. a) Juvenil. b) Adulto. II. Polimiositis: • Mediada por células $T(\alpha / \beta$, $\gamma / \delta)$ • Eosinofilica. III. Síndromes de superposición: • Con polimiositis. • Con dermatomiositis. - Con miositis asociada a neoplasias. - Con miositis por cuerpos de inclusión. IV. Miositis asociada a neoplasias. V. Miositis por cuerpos de inclusión. VI. Otras formas: • Focal: miositis orbitaria, nodular localizada, pseudotumor inflamatorio.

- Difusa: miofascitis macrofágica, miopatía necrotizante, miositis infantil.

Se aprecia en la indagación realizada, que aun cuando otros autores actualmente se han dedicado a investigar acerca de las clasificaciones de estas miopatías autoinmunes, tomando en cuenta sus formas cutáneas e infecciosas, y otros elementos de prevalencia; sigue predominando la realizada por Bohan y Peter en 1975, en la cual se determina de acuerdo a los síntomas evidenciados el tipo de enfermedad que presenta el sujeto.

\section{Causas}




\section{Factores que influyen en la aparición de dermatomiositis como enfermedad autoinmune}

Vol. 2, núm. 3., (2018)

Muso Pilchisaca Carol Yacqueline; Alex Christopher Ruiz Arriciaga; Cristhian Javier Córdova Molina; Mariuxi Jacqueline Coronel Reinoso

No se conoce del todo su etiología, se postula que factores genéticos y ambientales desempeñan un papel importante en el origen de la enfermedad. Se considera una condición de patogenia autoinmune, que se manifiesta por miopatía inflamatoria proximal en los músculos extensores y lesiones cutáneas patognomónicas. (Bohan, 1975).

A criterio de Nagaraju (2017) "la causa de la dermatomiositis se desconoce". Los expertos piensan que puede "deberse a una infección viral de los músculos o a un problema con el sistema inmunitario del cuerpo. Además, puede ocurrir en personas que tienen cáncer de abdomen, pulmón u otras partes del cuerpo". (p. 85). Destacando además que "cualquier persona puede desarrollar esta afección". (p.92).

Se presenta más a menudo en niños entre los 5 y los 15 años, y adultos entre 40 y 60 años. Esta enfermedad afecta con mayor frecuencia a las mujeres que a los hombres".

La causa de dermatomiositis según O'Callaghan (2008) "actualmente se desconoce; sin embargo, existen teorías sobre la interacción de agentes externos (ya sean físicos, químicos, neoplásicos o infecciosos), que provocan alteraciones autoinmunitarias cuando actúan en un territorio genético predispuesto". (p. 199).

Con relación a lo anterior, se deriva que el desconocimiento de una fuente sólida en cuanto a las causas que activan la dermatomiositis dificultan su detección y tratamiento en un tiempo apropiado que la enfermedad se presente totalmente; contrario a ello, alguno autores consideran que elementos de tipo infecciosos, genéticos y/o ambientales pudieran originarla: al 


\section{Factores que influyen en la aparición de dermatomiositis como enfermedad autoinmune}

Vol. 2, núm. 3., (2018)

Muso Pilchisaca Carol Yacqueline; Alex Christopher Ruiz Arriciaga; Cristhian Javier Córdova Molina; Mariuxi Jacqueline Coronel Reinoso

efecto es de gran importancia buscar aportes concluyentes y significativos que destaquen la manera de atacar oportunamente esta enfermedad autoinmune.

\section{Síntomas}

La forma más común de presentarse esta enfermedad autoinmune a juicio de Moreno (1999) es manifestada por "las lesiones de piel que representan el síntoma inicial de la enfermedad en el $50 \%$ de los pacientes y pueden preceder a la afección muscular en un período variable de tiempo, en el niño es frecuente que aparezcan a los pocos días o semanas del inicio de las manifestaciones musculares". (p. 400).

Arguellos (2007) señala que la dermatomiositis como una miopatía inflamatoria, "se reconoce por lesiones cutáneas, evolucionando con debilidad muscular progresiva y a menudo simétrica”. (p. 53). En un 15-20\% de los pacientes se presenta con disfagia. Ésta se asocia con deficiencias nutricionales, predisposición a neumonía por aspiración, disminución en la calidad de vida y mal pronóstico (Espinosa, 2010; p.523).

Las lesiones dérmicas se manifiestan por un eritema característico que acompaña (y más a menudo antecede) a la debilidad muscular. La erupción puede ser una zona hipercrómica de color azul-violáceo en los párpados superiores con edema (eritema en heliotropo); una erupción rojiza, plana en la cara y mitad superior del tronco; y eritema en los nudillos como una erupción violácea exfoliativa sobresaliente (signo de Gottron). (Zapata, 2009). 


\section{Factores que influyen en la aparición de dermatomiositis como enfermedad autoinmune}

Vol. 2, núm. 3., (2018)

Muso Pilchisaca Carol Yacqueline; Alex Christopher Ruiz Arriciaga; Cristhian Javier Córdova Molina; Mariuxi Jacqueline Coronel Reinoso

De tal manera, se puede deducir que la enfermedad es de detección tardía, dado que los síntomas aparecen de acuerdo a diversos factores de índole interna y/o externa y que pueden ser asociados con otros padecimientos, por tanto a pesar de que los síntomas señalados pueden determinar que se trata de la enfermedad, es necesario realizar un diagnostico con criterio preciso y mediante diversos exámenes complementarios para confirmar la presencia de dermatomiositis.

\section{Factores}

La literatura consultada, generalmente describe que la enfermedad autoinmune puede ser desencadenada por factores genético al efecto Feldman (2008) considera que "esos son susceptibles para la dermatomiositis" (p.209). En este sentido, los factores ambientales a criterio de Irazoque (2009) como "la radiación ultravioleta ocasiona apoptosis y exposición de autoantígenos en el exterior de la célula; así se favorece la pérdida de autotolerancia periférica y se inicia la cascada de autoinmunidad". (p. 03). Con respecto a los medicamentos que pueden causar miopatías como la dematomiositis "se encuentran productos de cosméticos, suplementos dietéticos, vacunas" (Cukier, 1993, p. 503).

Los factores mencionados según la literatura consultada, no constituyen resultados concluyentes que determinen la incidencia de estos en la aparición de la enfermedad, dado que los mismos son descritos de manera generalizada; sin embargo, se puede señalar que la que presenta la literatura como mayor influencia de generar la enfermedad es el factor genético siempre y cuando venga asociado con otros síntomas y elementos familiares del paciente que la padece. 


\section{Factores que influyen en la aparición de dermatomiositis como enfermedad autoinmune}

Vol. 2, núm. 3., (2018)

Muso Pilchisaca Carol Yacqueline; Alex Christopher Ruiz Arriciaga; Cristhian Javier Córdova Molina; Mariuxi Jacqueline Coronel Reinoso

La dermatomiositis es la enfermedad autoinmune considerada como rara, de tal manera que es poco frecuente la información en cuanto a factores que pueden crearla; la literatura generalmente resalta las causas, síntomas, y posibles tratamientos para sobrellevarla. No obstante a continuación se presenta una representación gráfica donde se aprecia los principales síntomas clínicos de la misma.

\section{Representación gráfica 1. Síntomas de Dermatomiositis}

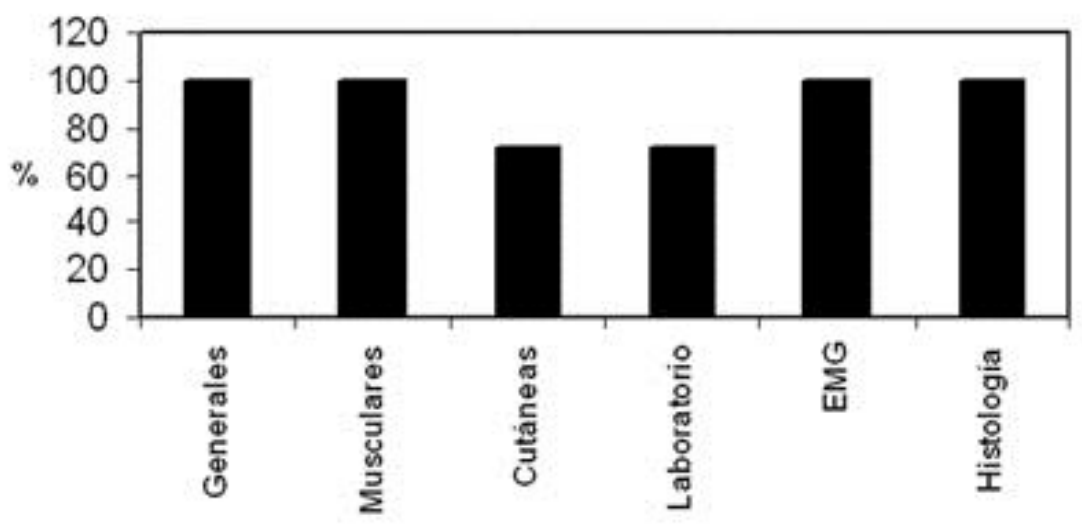

Fuente: Muñoz (2005). Manifestaciones clínicas, alteraciones de laboratorio, histológicas y en la EMG al diagnóstico en pacientes con dermatomiositis. Publicado por Muñoz B., Eliana. (2005). Experiencia de 10 años en dermatomiositis juvenil: A 10 -year-old experience. Revista chilena de pediatría, 76(4), 404-409.

El grafico exhibe la incidencia de los síntomas de dermatomiositis, siendo las más significativas síntomas musculares, EMG, histología. La literatura escasamente muestra datos 


\section{Factores que influyen en la aparición de dermatomiositis como enfermedad autoinmune}

Vol. 2, núm. 3., (2018)

Muso Pilchisaca Carol Yacqueline; Alex Christopher Ruiz Arriciaga; Cristhian Javier Córdova Molina; Mariuxi Jacqueline Coronel Reinoso

que puedan contrastar con la información de factores asociados a la enfermedad. Muñoz (2005)

presenta la tabla acerca de los criterios de Bohan

Tabla 1. Criterios de Diagnósticos de dermatomiositis según Bohan

\begin{tabular}{|c|c|c|}
\hline Criterios diagnósticos DMJ (Bohan-Peter) & $\begin{array}{l}\mathrm{N}^{*} \text { presente } \\
\text { al diagnóstico }\end{array}$ & $\begin{array}{l}\% \text { presente } \\
\text { al diagnóstico }\end{array}$ \\
\hline 1) Debilidad muscular proximal simètrica & $11 / 11$ & $100 \%$ \\
\hline $\begin{array}{ll}\text { 2) Compromiso cutảneo: } & \\
& \text { Total con compromiso cutáneo } \\
& \text { Rash heliotropo } \\
& \text { Pápulas de Gottion } \\
& \text { Otros }\end{array}$ & $\begin{array}{l}8 / 11 \\
6 / 11 \\
5 / 11 \\
5 / 11\end{array}$ & $\begin{array}{l}73 \% \\
54 \% \\
45 \% \\
45 \%\end{array}$ \\
\hline $\begin{array}{l}\text { 3) Elevación de enzimas musculares: } \\
\qquad \begin{array}{l}\text { LDH (UL) } \\
\text { CK (UL) }\end{array}\end{array}$ & $\begin{array}{l}4 / 11 \\
6 / 11\end{array}$ & $\begin{array}{l}36 \% \\
54 \%\end{array}$ \\
\hline 4) EMG: triada caracteristica observada en miositis & $9 / 9$ & $100 \%$ \\
\hline 5) Evidencias de inflamación crónica en biopsia muscular & $5 / 5$ & $100 \%$ \\
\hline
\end{tabular}

Fuente: Bohan (1975). Criterios diagnósticos de Bohan-Peter en pacientes con Dermatomiositis. Bohan A, Peter JB: Polymyositis and dermatomyositis. N Engl J Med 1975; 13: 344-7.

Representación gráfica 2. Evolución de manifestaciones clínicas y exámenes de laboratorio en pacientes con dermatomiositis 


\section{Factores que influyen en la aparición de dermatomiositis como enfermedad}

autoinmune

Vol. 2, núm. 3., (2018)

Muso Pilchisaca Carol Yacqueline; Alex Christopher Ruiz Arriciaga; Cristhian Javier Córdova

Molina; Mariuxi Jacqueline Coronel Reinoso

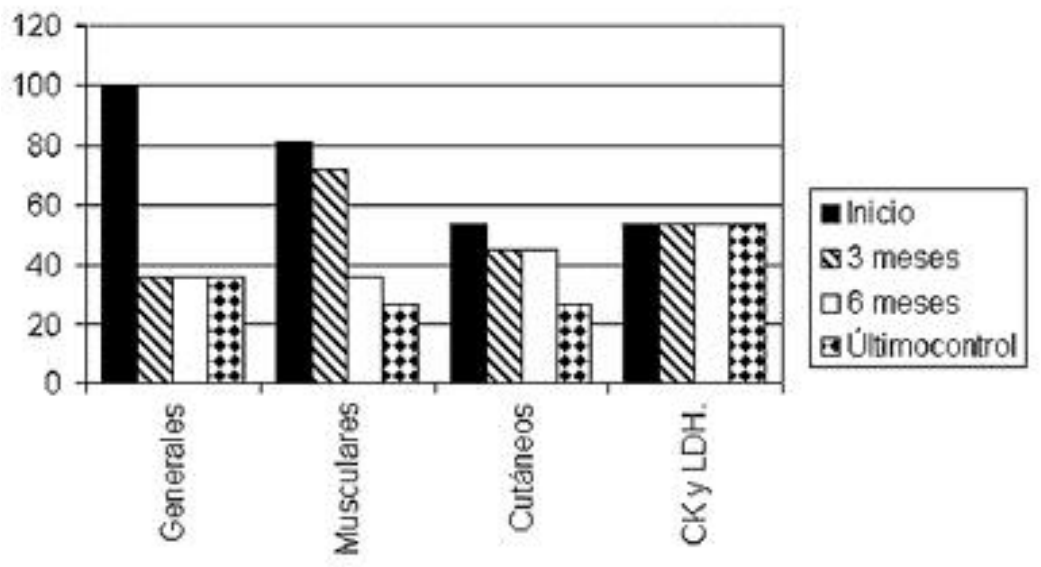

Fuente: Muñoz (2005). Evolución de manifestaciones clínicas y exámenes de laboratorio en 11 pacientes con dermatomiositis.. Publicado por Muñoz B., Eliana. (2005). Experiencia de 10 años en dermatomiositis juvenil: A 10 -year-old experience. Revista chilena de pediatría, 76(4), 404409.

Puede derivarse de estos resultados, que se tiene solo información de los síntomas encontrados de teorías de manera obsoleta en la literatura; destacándose los síntomas tanto cutáneos como musculares de la enfermedad, así como los criterios para determinarla, los cuales se apoyan en exámenes de laboratorios. Lo anterior conlleva reflexionar que se necesita más investigaciones relacionadas con los factores de incidencia de la enfermedad.

\section{Conclusiones.}

Como enfermedad autoinmune, la dermatomiositis constituye hoy día, una miopatía inflamatoria de gran repercusión en la sociedad, lo cual deriva en la significación de efectuar estudios sobre la misma donde pueda generarse información acerca de su incidencia por cuanto 


\section{Factores que influyen en la aparición de dermatomiositis como enfermedad autoinmune}

Vol. 2, núm. 3., (2018)

Muso Pilchisaca Carol Yacqueline; Alex Christopher Ruiz Arriciaga; Cristhian Javier Córdova Molina; Mariuxi Jacqueline Coronel Reinoso

actualmente es poca la información que se tiene sobre ella, y aportar tratamiento para la enfermedad.

Las miopatías autoinmunes en general han sido objeto de estudio, no obstante la dermatomiositis poco ha tenido soporte de información que pueda dar significatividad en relación a la amenaza que presenta para la salud y vida del individuo.

En la investigación se concluye que los factores que tiene mayor incidencia en la prevalencia de esta enfermedad son diversos y no son concluyentes; solo se muestran teorías acerca de la posible vinculación de factores externos diversos que pudieran activar la enfermedad. De acuerdo a la literatura citada y a pesar de que no se tiene un definitivo factor de incidencia, se infiere que la dermatomiositis presenta tratamiento para su estabilidad por largos lapsos de tiempo.

\section{Referencias}

Amato AA, Barohn RJ. Evaluation and treatment of inflammatory myopathies. J Neurol Neurosurg Psychiatry. 2009;80:1060-8.

Arguello Ruíz,M. Baumgartner Reyes. Dermatomiositis. Revista Médica de Costa Rica y Centroamérica, 64 (2007), pp. 53-55

Bielsa-Marsol I. Dermatomiositis. Reumatol Clin. 2009; 5 (5): 216-222.

Bohan, A. Peter Jb.Dermatomyositis. Engl J Med. 1975; 292: 344-743.

Cukier, J. Beauchamp RA, Spindler S. Lorenzo C. Association Between bovine collagen dermal implants and a dermatomyositis or a polymiositis- like syndrome. 1993. 118: 920-928.

Dalakas MC, Hohlfeld R. Polymyositis and dermatomyositis. Lancet. 2003;362:971-82

Dalakas. M.C. Inflammatory myopathies. Curr Opin Neurol Neurosurg, 3 (1991), pp. 689-696

Revista Científica Mundo de la Investigación y el Conocimiento. 2 (3). pp. 510-526 


\section{Factores que influyen en la aparición de dermatomiositis como enfermedad autoinmune}

Vol. 2, núm. 3., (2018)

Muso Pilchisaca Carol Yacqueline; Alex Christopher Ruiz Arriciaga; Cristhian Javier Córdova Molina; Mariuxi Jacqueline Coronel Reinoso

Espinoza-Cobos, J. Pérez-figueroa,G. Zuñiga-Ahuet,M.A. Dorantes Disfagía orofaríngea como primera manifestación de dermatopolimiositis asociada a cáncer de colon. Rev Gastroenterol Mex, 75 (2010), pp. 522-527

Feldman B, Rider L, Reed A, Pachman L. Juvenile dermatomyositis and other idiopathic inflmmatory myopathies of childhood. Lancet 2008; 371: 201-212.

Fernández, E. Dermatomiositis. XII Congreso Médico Nacional. Revista Médica Hondureña. 2da Epoca. Servicio de Dermatologia. Clinica Facultad de Medicna. Honduras. 1967. Pp.1317.

Gerami P, Schope JM, McDonald L, Walling HW, Sontheimer RD. A systematic review of adult-onset clinically amyopathic dermatomyositis (dermatomyositis sine myositis): a missing link within the spectrum of the idiopathic infl ammatory myopathies. J Am Acad Dermatol. 2006; 54 (4): 597-613.

Hassan ML, Capecce A, Melloni ME, Saposnik M, Ruzzi I, Gramajo J et al. Estudio retrospectivo de la dermatomiositis. análisis de 40 casos de consulta en la División de Dermatología. Dermatol Argent. 2009; 15 (1): 27-36.

Irazoque PF. Barragan, NY. Epidemiologia, etiología y clasificación. Reumatol Clib. 2009; 5 (3): $2-5$.

Karpati,S. G. Carpenter. Idiopathic inflammatory myopathies. Curr Op Neurol Neurosurg, 1 (1988), pp. 806-814

Mastaglia F.L., Phillips B.A. Idiopathic inflammatory myopathies: Epidemiology, classification, and diagnostic criteria. Rheum Dis Clin North Am. 2002;28:723-41.

Moreno P., Martínez CN, Oliva S. Dermatomiositis juvenil. En González E. Manual Práctico de Reumatología Pediátrica. Madrid. Laboratorios Menarini.1999. 397-418 Moreno P., Martínez CN, Oliva S. Dermatomiositis juvenil. En González E. Manual Práctico de Reumatología Pediátrica. Madrid. Laboratorios Menarini.1999. 397-418

Muñoz B., Eliana. (2005). Experiencia de 10 años en dermatomiositis juvenil: A 10 -year-old experience. Revista chilena de pediatría, 76(4), 404-409.

Jacobson DL, Gange SJ, Rose NR, Graham NMH. Epidemiology and estimated population burden of selected autoimmune diseases in the United States. Clin Immunol Immunopathol. 1997;84(3):223- 43. 


\section{Factores que influyen en la aparición de dermatomiositis como enfermedad autoinmune}

Vol. 2, núm. 3., (2018)

Muso Pilchisaca Carol Yacqueline; Alex Christopher Ruiz Arriciaga; Cristhian Javier Córdova

Molina; Mariuxi Jacqueline Coronel Reinoso

Nagaraju K, Gladue HS, Lundberg IE. Inflammatory diseases of muscle and other myopathies. In: Firestein GS, Budd RC, Gabriel SE, McInnes IB, O'Dell JR, eds. Kelley and Firestein's Textbook of Rheumatology. 10th ed. Philadelphia, PA: Elsevier; 2017:chap 85.

O’Callaghan A. Trallero, E. Mioppaias inflamatorias. Dermatomiositis, poliomisitis y miositis con cuerpos de inclusión. Reumatol Clin. 2008.; 4: 197-206

Peloro T, Miller F, Hahn T, Newman E: Juvenile Dermatomiositis: a retrospective review of 30 year experience. J Am Acad Dermatol 2001; 45: 28-34.

Vidarte Orrego G, Alvarez Llanos E, Lozano Miranda Z. Dermatomiositis amiopática: reporte de un caso y revisión de la literatura. Dermatol Peru. 2008; 18(1): 45-50.

Villa-Juárez M. Ricardo Y, Polimiositis/ dermatomiositis y embarazo. Rev. SaludmILITAR Mex. 2002;56(1):40-45.

Zapata, A.F. Ramírez. Manifestaciones cutáneas de las neoplasias malignas. Rev Asoc Col Dermatol, 17 (2009), pp. 109-120 